\title{
Does Bike Sharing increase House Prices? Evidence from Micro-level Data and the Impact of COVID-19
}

\author{
Zhengyi Zhou' ${ }^{1} \cdot$ Hongchang $\mathrm{Li}^{2} \cdot$ Anming Zhang $^{3}$
}

Accepted: 25 January 2022

(c) The Author(s), under exclusive licence to Springer Science+Business Media, LLC, part of Springer Nature 2022

\begin{abstract}
With unique datasets, this paper studies the effects of dockless bike sharing on house prices. We find that in neighborhoods relatively far from subway stations, house prices increase with the usage intensity of shared bikes. This indicates a positive value of bike sharing as a complement to the subway network. Meanwhile, shared bike usage intensity also has a negative impact on house prices. The negative effect is mitigated for luxury neighborhoods and neighborhoods near City Management Teams, suggesting that the negative effect is related to bike misplacement. Since the breakout of COVID-19, both the positive and negative price impacts have become more evident. This is consistent with the fact that the user base of shared bikes, which allow for social distancing in an open space, has increased during the pandemic. This may enhance people's confidence in the long survival of the bike sharing industry.
\end{abstract}

Keywords Dockless bike sharing $\cdot$ Subway $\cdot$ House price $\cdot$ COVID-19 China

JEL R42 $\cdot$ P25 $\cdot$ D62

Zhengyi Zhou

zhou.zhengyi@mail.sufe.edu.cn

Hongchang Li

hchli@bjtu.edu.cn

Anming Zhang

anming.zhang@sauder.ubc.ca

1 School of Finance, Shanghai University of Finance and Economics, Yangpu District, 100 Wudong Road, Shanghai 200433, China

2 School of Economics and Management, Beijing Jiaotong University, Haidian District, No.3 Shangyuancun, Beijing 100044, China

3 Sauder School of Business, University of British Columbia, Vancouver, BC V6T1Z2, Canada 


\section{Introduction}

As a healthy and sustainable transportation mode, bike sharing has become popular in thousands of cities around the world since its first appearance in Amsterdam in the 1960s (e.g., Gu et al., 2019). Bike sharing companies are predominantly privately run, and there has been much discussion in the popular press on welfare impacts of this industry in recent years. But in the literature, the welfare impacts remain under-explored.

The welfare impact of the bike sharing industry is complicated. On the positive side, bike sharing utilizes sharing-economy platforms, a business model that has gained momentum in urban areas around the world. Moreover, by solving the "last mile" problem, bike sharing improves people's access to the subway network. On the negative side, users of dockless shared bikes may misuse the scarce public space, such as parking a shared bike at an improper location. The net impact likely varies across different locations, depending on the local amenity.

In this paper, we examine the effect of bike sharing on house prices. House prices are routinely used to evaluate welfare benefits of local public goods (Teulings et al., 2018). Bike sharing is not a public good, but due to its "house externality" (RossiHansberg et al.I, 2010), we can still shed some light on its welfare effect by examining its house price impacts. We assume that the welfare gains and losses caused by shared bikes, which are not fully internalized by bike sharing companies, are capitalized into local house prices. This approach also allows us to observe the heterogeneity of bike-sharing effects across neighborhoods, which helps us find proper ways to increase the overall impact of the industry. ${ }^{1}$

We use Shanghai, China, as our research setting for two reasons. First, there is a large number of bike sharing users there, so the economic impacts are potentially large. By August 2019, the bike sharing industry had 300 million users in China. It covered 360 cities, with a daily rider volume of 47 million. ${ }^{2}$ This makes China the largest bike sharing market in the world (e.g., Gu et al., 2019). Shanghai is one of the first Chinese cities to introduce dockless bike sharing. Second, the benefits and costs of bike sharing are both obvious in Shanghai. On the one hand, Shanghai has the world's largest rapid transit (subway) system by route length (Zhou et al., 2021). Since bikes are not allowed on subways, the value of bike sharing as a complement to the public transportation network is potentially high. On the other hand, dockless bike sharing may decrease the general welfare of the public by eroding streets and walking spaces (Yin et al., 2019). This incurs a high social cost, especially given that Shanghai has very high population density and crowded streets.

Our micro-level datasets consist primarily of three parts. The first part is the riding records of Meituan Bike. Once known as Mobike, Meituan Bike is the first bike sharing brand in Shanghai, and one of the first brands in China. It has maintained the status as a major player in the bike sharing industry so far, accounting for about

\footnotetext{
${ }^{1}$ In Chinese cities, "neighborhood" is a small geographic unit with a clear boundary. A neighborhood consists of a cluster of houses built by the same developer on a piece of residential land. In our context, a "house" or "house unit" refers predominantly to an apartment unit. The median neighborhood has 34 apartment buildings.

${ }^{2}$ See China Consumer Journal on 8 January 2020. Established in 1985, the Journal is a major business newspaper in China.
} 
half of the market share. ${ }^{3}$ The second part is house listing data from Lianjia, one of the largest real estate brokerages in China. The third part is point-of-interest (POI) data from Baidu Map and AMap, both of which are Chinese counterparts of Google Map.

With the datasets, we estimate the net welfare benefit of bike sharing in the following steps. First, we calculate the intensity of Meituan Bike usage around each neighborhood. The calculation is based on the number of rides around a neighborhood during rush hours on 9 October 2017, which was 18 months after the launch of Meituan Bike in Shanghai. We assume that the intensity of shared bike usage on this day is representative for the steady state. The results are similar if we use the riding records in 2019 or 2020 to calculate the intensity. Second, we examine the relationship between house prices and the intensity of shared bike usage. If bike sharing has a positive value by complementing the subway network, then the house price gradient of subway stations should become flatter after the launch of Meituan Bike, and this pattern should be driven by the neighborhoods where shared bikes are heavily used. If bike sharing brings a negative external effect, then this should be more significant for neighborhoods without good property management service or public management service nearby. Finally, we investigate how COVID-19 has changed the relationship between bike sharing and house prices. As an unprecedented global pandemic, COVID-19 has reshaped people's travelling behavior. It is interesting to investigate whether the usage of shared bikes has increased or decreased during the pandemic, and whether the house price effects of bike sharing have become stronger or weaker.

Our findings are as follows. First, for neighborhoods that are relatively far away from subway stations (at least $1.2 \mathrm{~km}$ ), the relationship between house price and steady-state shared bike usage intensity has become more positive after the launch of Meituan Bike. This indicates a positive value of bike sharing as a complement to the subway network. Meanwhile, the shared bike usage intensity also has a negative impact on house prices. The negative impact is weaker for luxury neighborhoods and neighborhoods close to City Management Teams (CMTs). Sponsored by the government, CMTs are responsible for keeping the public area clean and tidy, including rearranging shared bikes and regulating the behavior of riders. Hence, the results suggest that the negative external effect of bike sharing is related to the misplacement of dockless shared bikes. In the vicinity of subway stations, where people can reach the stations by walking and the positive value of bike sharing as a complement to the subway network is virtually zero, the net house price effect of bike sharing is negative.

Second, after the breakout of COVID-19, both the positive value and the negative external effect of bike sharing have been more heavily capitalized into

\footnotetext{
3 We refer to the 2017 wave of China Household Finance Survey (CHFS). It covers 170 cities from 29 provinces of China, and includes a section about shared bike usage. Totally 1,453 urban respondents disclosed that they were shared bike users. Among them, 804 chose Meituan Bike, which represented 55\% of the shared bike users. The percentage for Shanghai is also 55\%. Please see more details about CHFS in Gan, Yin, Jia, Xu, Ma, and Zheng (2014).
} 
house prices. This is consistent with the fact that the user base of Meituan Bike has increased during the pandemic. The enhanced user base improves people's confidence about the long survival of the bike sharing industry, which strengthens its house price impacts.

The contribution of this paper lies in several aspects. First, this paper is among the first studies to quantify both the positive value and the negative external effect of shared bikes, within the context of an emerging economy. In particular, our evidence for the negative external effect is new to the literature. Second, to our best knowledge, this paper is the first study to examine the impact of a pandemic on the relationship between house price and bike sharing.

The rest of the paper is arranged as follows. The next section reviews the literature. Then we develop the hypotheses and present our empirical strategy. Following that, we provide the background knowledge of the bike sharing industry in Shanghai, and introduce our data. Next, we display our major empirical results. Finally, we conduct various robustness checks, and concludes.

\section{Literature Review}

In recent years, there is an emerging literature on bike sharing, which is a major player in the shared economy. With data from Pittsburgh, USA, Pelechrinis et al. (2017) find that the bike-sharing system led to an increase in housing prices in zip codes where shared bike stations were installed. Unlike them, we consider dockless shared bikes. Moreover, we conduct a house-level analysis, which enables us to explore the underlying mechanisms through which bikesharing affects house prices. Li and Joh (2017) find that jointly enhancing bikeability (e.g. bike sharing) and transit accessibility can generate positive synergistic effects on property values. Chu et al. (2021) estimate that the entry of dockless bike sharing reduces the subway premium in house prices by about one-third. The above two papers highlight the positive value of bike sharing as a complement to the subway system. That is, in the past, the accessibility of subway stations mainly depended on the walking environment (Li et al., 2017). But now, bike sharing has become an alternative to walking. Our paper is different from these two papers in several aspects. First, we make good use of the riding records of a major bike sharing company in China. This unique dataset enables us to explore how both the positive value and the negative external effect of dockless shared bikes vary across neighborhoods. Second, by including a sample period after the breakout of COVID-19, we investigate whether these values of bike sharing have been reduced or enhanced by the pandemic, which obviously affected people's transportation mode. 


\section{Hypothesis Development and Econometric Approach}

Since the seminal contributions of Alonso (1964), Muth (1969), Mills (1972) and others, the relationship between housing prices and the monetary and time costs of commuting has been at the core of urban economics (Theisen and Emblem, 2021). We use this stream of literature as our theoretical base. In particular, Theisen and Emblem (2018) study the relationship between house price and proximity to kindergarten. They propose that the proximity brings both convenience and disutility. Their model predicts that house prices increase with the distance to kindergarten when the distance is short. When the distance becomes longer, house prices decrease with the distance, but this negative relationship becomes weak when the distance is very long.

Like kindergarten, proximity to subway stations also brings both convenience and disutility. The convenience lies in the time savings brought by subways. Using subway Line 6 in Shanghai as an example, Zhou et al. (2021) show that the subway reduces residents' commuting time to major employment centers, which leads to house price appreciation in the vicinity of the subway. The local demand for subway service is large. According to National Bureau of Statistics (NBS) of China, the passenger volume of Shanghai subways was 3.9 billion in 2019, which translates into 160 rides per resident per year. On the other hand, subways may also bring disutility because of the noise and congestion around subway stations. Hence, the model of Theisen and Emblem (2018) provides a perfect theoretical guide for our study.

More specifically, their model shows that a falling commuting cost per kilometer, in terms of both time and money, would make the relationship between house price and distance to kindergarten less negative. In our setting, bike sharing reduces the generalized cost of commutes to subway stations, especially for residents living relatively far from subway stations. ${ }^{4}$ Assuming that the value of such improvement is not fully internalized by bike sharing companies, the "subway premium" in house prices should drop. This pattern would be stronger in places with a high intensity of shared bike usage. Thus, we have the following testable hypothesis:

H1: After the launch of Meituan Bike, the subway premium drops, and the drop concentrates in the neighborhoods where shared bikes are intensively used.

Despite of the positive value described above, bike sharing may generate a negative value as well, which lies in the utility loss of local residents, especially nonusers. For example, dockless sharing bikes are often misplaced and block the street (Yin et al., 2019). According to the Survey of Shared Bikes conducted by Shanghai Almanac in 2017, 75.4\% of the respondents regarded the misuse of public space as the most serious problem of dockless bike sharing.In some cases, the negative

\footnotetext{
${ }^{4}$ For the residents who used to walk from home to subway stations, bike sharing reduces their time cost, as long as the distance between home and station is not extremely short. For the residents who used to take a bus or taxi (or any other on-demand ride services) from home to subway stations, bike sharing reduces their monetary cost and/or time. Monthly bike-sharing membership sells for 16.8 yuan, which roughly equals the total fees of taking a bus for 8 times, or the minimum fee of taking a taxi for 1 time. Please see more details about the pricing of bike sharing service in the data description section. Furthermore, both the bus and taxi services generally involve more waiting time for users than bike sharing.
} 
external effect caused by the misplacement of shared bikes may even outweigh the potential benefits. For example, when one's home is very close to a subway station, the "last mile" problem is largely absent. Then the positive value would be dominated by the negative external effect. Assuming that the negative external effect increases with the intensity of shared bike usage, we have the following testable hypothesis:

H2: For neighborhoods close to subway stations, house prices decrease with the local intensity of shared bike usage.

To examine $\mathrm{H} 1$ and $\mathrm{H} 2$, our baseline regression is designed as shown in formula (1) below. The sample period covers from 2011 to 2019. The regression tests how the house price effects caused by the launch of Meituan Bike vary with the intensity of shared bike usage. In this sense, we adopt the difference-in-differences approach (Angrist and Pischke, 2009), which is by now a standard approach used in the literature on the house price impacts of various infrastructures (e.g. Theisen and Emblem, 2021).

The dependent variable is the natural logarithm of the transaction price of house $i$, which belongs to neighborhood $n$ in zone $z$ and was transacted in month $t$. On the right-hand side, the key variable $\ln B i k e$ is a neighborhood-level variable that measures the steady-state intensity of shared usage; the detailed definition will be given in the data description section. Post is a dummy that equals 1 for dates after 22 April 2016 (the date when Meituan Bike was launched in Shanghai), and 0 for earlier dates. $D S u b$ is the distance to the nearest subway station.

$$
\begin{aligned}
& \ln \text { Prc }_{i, n, z, t}=c+\beta_{1} \ln \text { Bike }_{n}+\beta_{2} \ln \text { Bike }_{n} * \text { DSub }_{n}+\beta_{3} \text { DSub }_{n}+\beta_{4} \ln \text { Bike }_{n} * \text { Post }_{t} \\
& +\beta_{5} \ln \text { Bike }_{n} * \text { DSub }_{n} * \text { Post }_{t}+\beta_{6} \text { DSub }_{n} * \text { Post }_{t}+\beta_{7} \text { ManaFee }_{n}+\beta_{8} \text { DCenter }_{n}+\beta_{9} \text { Size }_{i} \\
& +\beta_{10} \text { Room }_{i}+\beta_{11} \text { Parlor }_{i}+\beta_{12} \text { Position }_{i}+\beta_{13} \text { TotFloor }_{i}+\beta_{14} \text { Position }_{i} * \text { TotFloor }_{i} \\
& +\beta_{15} \text { Age }_{i}+\lambda_{i}^{F}+\lambda_{i}^{D}+\lambda_{i}^{B}+\mu_{z}+\tau_{t}+\varepsilon_{i, n, z, t}
\end{aligned}
$$

If $\mathrm{H} 1$ is true, we should observe a positive coefficient of $\ln B i k e^{*} D S u b^{*}$ Post. That is, after the launch of Meituan Bike, the decrease in subways premium should be larger in neighborhoods where shared bikes are intensively used.

If $\mathrm{H} 2$ is true, we should observe a negative coefficient of $\ln B i k e^{*}$ Post. This coefficient reveals the general effect that applies to all houses. It tells us what the effect of bike sharing is like if we control for its positive value as a complement to the subway network by $\ln B i k e^{*} D S u b^{*}$ Post. The coefficient can also be regarded as an estimation for the effect on houses with a zero distance from their nearest subway stations.

Our dataset allows us to include a rich set of controlling variables in the regression. First, we control for house characteristics, such as size (Size), the number of bedrooms (Room), the number of parlors (Parlor), and the position of the house in an apartment building (Position). And we control for the direction to which the house faces and the level of interior decoration by two vectors of dummies, $\lambda^{F}$ and $\lambda^{D}$, respectively. Second, we control for building characteristics like age (Age) and the total number of floors (TotFloor). The apartment buildings constructed in the 1970s or earlier typically have 7 or fewer floors and have no elevators. High-rise apartment buildings have elevators and are newer. We also include an interaction 
between Position and TotFloor because low-rise buildings do not have elevators and thus higher levels are less favorable. For high-rise buildings, in contrast, higher levels are more favorable because of the nice view. And we control for building type by a vector of dummies, $\lambda^{B}$; slab-type buildings are usually more favorable than tower-type buildings. ${ }^{5}$ Third, we control for neighborhood characteristics such as the management fee charged by the property management company (ManaFee) and the distance to the city center, i.e., People's Square (DCenter). The detailed definitions of the major variables used in this paper are displayed in Appendix A. Finally, we control for zone fixed effects. In addition to subway stations, other amenities also matter (e.g. Des Rosiers et al., 2001), but it is impossible for us to control for all amenity factors. Lianjia's definition of "zone" is based on local amenity; neighborhoods in the same residential zone have similar amenity. Hence, by controlling for zone fixed effects, we alleviate the omitted variable problem. We also control for year-month fixed effect by dummies, which capture the time variation in the general house price level.

To further identify the source of the negative external effect, we investigate its heterogeneity across neighborhoods. If it is caused by bike misplacement, this problem should be alleviated in places where the public space is carefully managed. For example, luxury neighborhoods usually purchase high-quality property management service. The service includes maintaining a clean and orderly environment in and around the neighborhood, which may mitigate or even eliminate the bike misplacement problem. Another example is City Management Teams (CMTs). Hired by the government, CMTs are responsible for public space in open areas, such as streets. Consequently, the neighborhoods near the streets may benefit from their work. Then, we have a hypothesis related to $\mathrm{H} 2$ :

H2a: For the neighborhoods near subway stations, the negative relationship between shared bike usage and house price is driven by non-luxury neighborhoods and neighborhoods relatively far from CMT offices.

We use subsample analysis to examine H2a. We first test whether the negative coefficient of $\ln B i k e^{*}$ Post has a larger magnitude if we exclude houses in luxury neighborhoods. Then, we divide houses into two groups according to their distance to the nearest CMT office, and examine whether this negative coefficient has a larger magnitude for houses that are relatively far from CMT offices.

The breakout of COVID-19 has a large influence on people's transportation modes (e.g., Zhang et al., 2020; Eisermann et al., 2021). To understand the impact of such a pandemic on the relationship between bike sharing and house prices, we conduct further analysis. On the one hand, alternative public transportation modes like buses may be replaced with bike sharing, which allows for social distancing between users. This may result in a larger user base of the bike sharing industry, which in turn will enhance people's confidence in the long survival of this industry.

\footnotetext{
5 We have 4 direction categories: East, South, West, and North. If a house faces, for example, southeast, then the dummies South and East both equal 1. We have 4 decoration categories: High, Medium, No decoration, and Unknown. We have 5 building type categories: Slab, Tower, Hybrid, Bungalow, and Unknown.
} 
Accordingly, its house price impacts will be strengthened. On the other hand, the pandemic leads to a temporary drop in the demand for subways because of their high passenger density. Consequently, the value of bike sharing as a complement to the subway system may decrease. As the usage of shared bikes drops, the misplacement problem will naturally be alleviated. Therefore, it is an empirical question whether the pandemic strengthens or weakens the house price effects of bike sharing. We have the following testable hypothesis:

H3: COVID-19 strengthens the relationships between shared bike usage and house prices.

To examine H3, we extend our sample period to 13 July 2020. We define PostCOVID as a dummy that equals 1 for dates after 1 January 2020, and 0 for earlier dates. Wuhan was locked down on 23 January 2020, but the news about the epidemic actually came out at the beginning of January 2020. Then we add three interaction terms into regression (1): $\ln B i k e^{*}$ PostCOVID, $\ln B i k e^{*}$ DSub*PostCOVID, and $D S u b^{*}$ PostCOVID. If $\mathrm{H} 3$ is true, we should observe that the coefficient of $\ln B i k e^{*}$ PostCOVID is significantly negative, and the coefficient of $\ln B i k e^{*} D S u b^{*}$ PostCOVID is significantly positive.

\section{Background and Data Description}

This section first provides the background knowledge of the bike sharing industry in Shanghai, China. Then, we describe the data used to test the hypotheses.

\section{Bike Sharing in Shanghai, China}

In China, dockless bike sharing is operated by private firms. Although these firms are not state-owned, they receive government support in that dockless shared bikes use public space freely. The bike-sharing market is highly concentrated. Once known as Mobike, Meituan Bike has been one of the oligopolists since its launch. According to Sohu News (a major news portal in China), it became the largest bike sharing company of China in May 2017. ${ }^{6}$

Shanghai have three major players in the bike sharing market: Meituan Bike, Hellobike, and Didi bike. Meituan Bike is the earliest one. It entered Shanghai on 22 April, 2016. This was six months earlier than the entry of Ofo, which was another major bike-sharing brand but defaulted later. ${ }^{7}$ Meituan Bike offers a variety of service packages. For example, the 7-day, 30-day, and 90-day membership cards are sold for 5.8 yuan (\$0.9), 16.8 yuan (\$2.6), and 49 yuan (\$7.5), respectively. Daily users usually buy 30-day or 90-day membership cards. For one-time users, Meituan Bike charges a fee of 1.5 yuan for the first $15 \mathrm{~min}$, and 0.5 yuan for each additional

\footnotetext{
6 See: http://business.sohu.com/20170517/n493354558.shtml

7 Hellobike first started its business in small and medium cities; it did not enter into first-tier cities like Shanghai until 2018. Didi Bike was also launched in 2018. Therefore, using Meituan Bike data allows us to have a clean pre-launch period (i.e. no bike sharing service of any brand in that period) and a long post-launch period.
} 


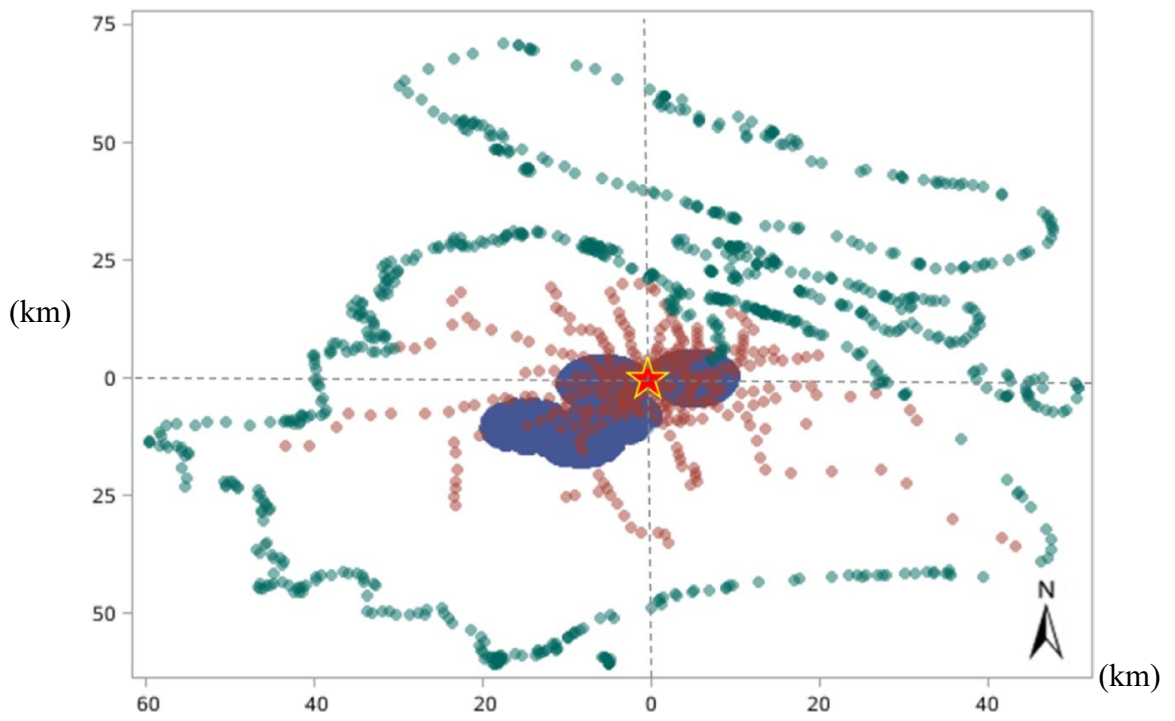

Fig. 1 Sample area. Note. The blue region is our sample area. The red star marks the $(0,0)$ point, which is the city center, i.e. People's Square. The red dots are subway stations. The green dots show the boundary of Shanghai. The $\mathrm{x}$-axis and the $\mathrm{y}$-axis shows the distance $(\mathrm{km})$ to the city center, respectively

15 min. In general, relative to bus service, bike sharing service is cheap; buses charge 2 yuan per passenger. ${ }^{8}$

\section{Data}

This section introduces three micro-level datasets that we combine together: Meituan Bike riding records, transaction records of individual houses, and POI information.

\section{Meituan Bike Riding Records}

Figure 1 shows the location of our sample area relative to the subway network of Shanghai. All the rides in our sample started from the sample area, and the ending points can be anywhere. The sample area consists of five regions, and the radius of each region is $5 \mathrm{~km} .{ }^{9}$ The five regions involve the inner-ring area, the middle-ring

\footnotetext{
${ }^{8}$ During the sample period 2011-2019, the exchange rate between the Chinese currency and the U.S. dollar is 6.5 yuan per dollar. The data source is Wind database, which is a Chinese counterpart of Bloomberg and widely used in the financial industry.

${ }^{9}$ Meituan Bike allowed us to choose five regions, with each region having a radius of no more than $5 \mathrm{~km}$. We decide to choose five regions with important subway stations. We try to make a balance between the passenger volume of the stations and the density of residents nearby. We also try to avoid choosing overlapping regions so as to maximize the geographic coverage. Finally, we choose five regions, the centers of which are five important subway stations; Shanghai has more than 300 subway stations, and the five stations have the $7^{\text {th }}, 13^{\text {th }}, 10^{\text {th }}, 4^{\text {th }}$, and $5^{\text {th }}$ largest passenger volume in 2015, according to our data of transportation card usage record. Some may be concerned that $\ln B i k e$ of neighborhoods at the edge of the sample area may contain measurement errors and bias our results. Hence, we try alternative radius ranging from 1 to $4 \mathrm{~km}$. The baseline regression results are similar and available upon request.
} 


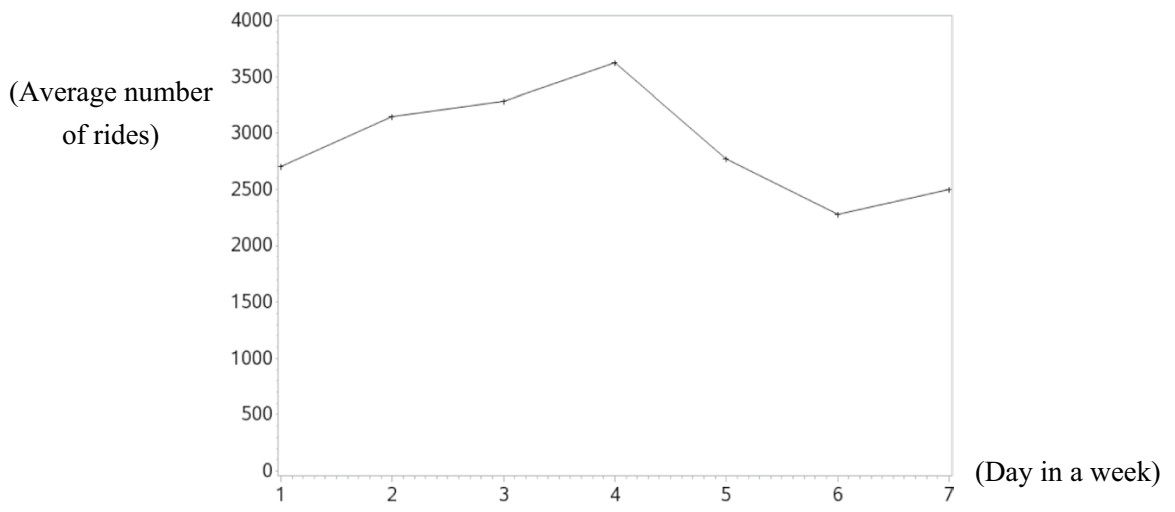

Fig. 2 Number of rides on weekdays and weekends. Note. The figure shows the average number of rides on Monday through Sunday. The X-axis shows the day in a week. For example, "1" refers to Monday. Holidays that coincide with Monday to Friday are excluded. The sample period is May to June, 2016

area, the outer-ring area, and the area outside the outer-ring. ${ }^{10}$ Hence, our sample area contains rich spatial variation.

Although the sample area does not seem large relative to the whole city, it contributes up to $37 \%$ of the house transactions done through Lianjia from 2011 to 2020. The "nearest subway stations" of these houses represent $41 \%$ of all the subway stations in Shanghai; these stations are located in the central part of the subway network and have large passenger flows. The high density of residents in the sample area provides a good setting for the shared economy. The high density also means a potentially large demand for subway service; with evidence from Beijing, China, Li (2020) shows that transit accessibility offers little travel advantage outside of dense urban areas. Hence, we expect the positive value of bike sharing to be large in our sample area.

We have the riding records of five periods: May 2016, June 2016, 9 October 2017, 9 October 2019, and 9 June 2020. May and June of 2016 are the first two months after the launch of Meituan Bike, and the season is suitable for riding. In our sample areas, there were 52,078 rides and 158,147 rides in May and June of 2016, respectively. With the riding records in the two months, we discover interesting riding patterns of Meituan Bike users. For example, as can be seen from Fig. 2, there are more rides on weekdays than on weekends, with the largest number of rides on Thursday. Moreover, on weekdays, the number of rides peaks at 8:00-9:00 and 18:00-19:00, as shown in the upper panel of Fig. 3. This "dual peaks" pattern of the morning and evening rush hours indicates that commuting is an important purpose of using shared bikes. On the weekend, on the other hand, the number of rides peaks at one period (16:00-18:59), as can be seen in the lower panel.

10 Shanghai is divided into four parts by the inner-ring, middle-ring, and outer-ring. The area inside the inner-ring is the traditional downtown. The area outside the outer-ring is the suburb. 


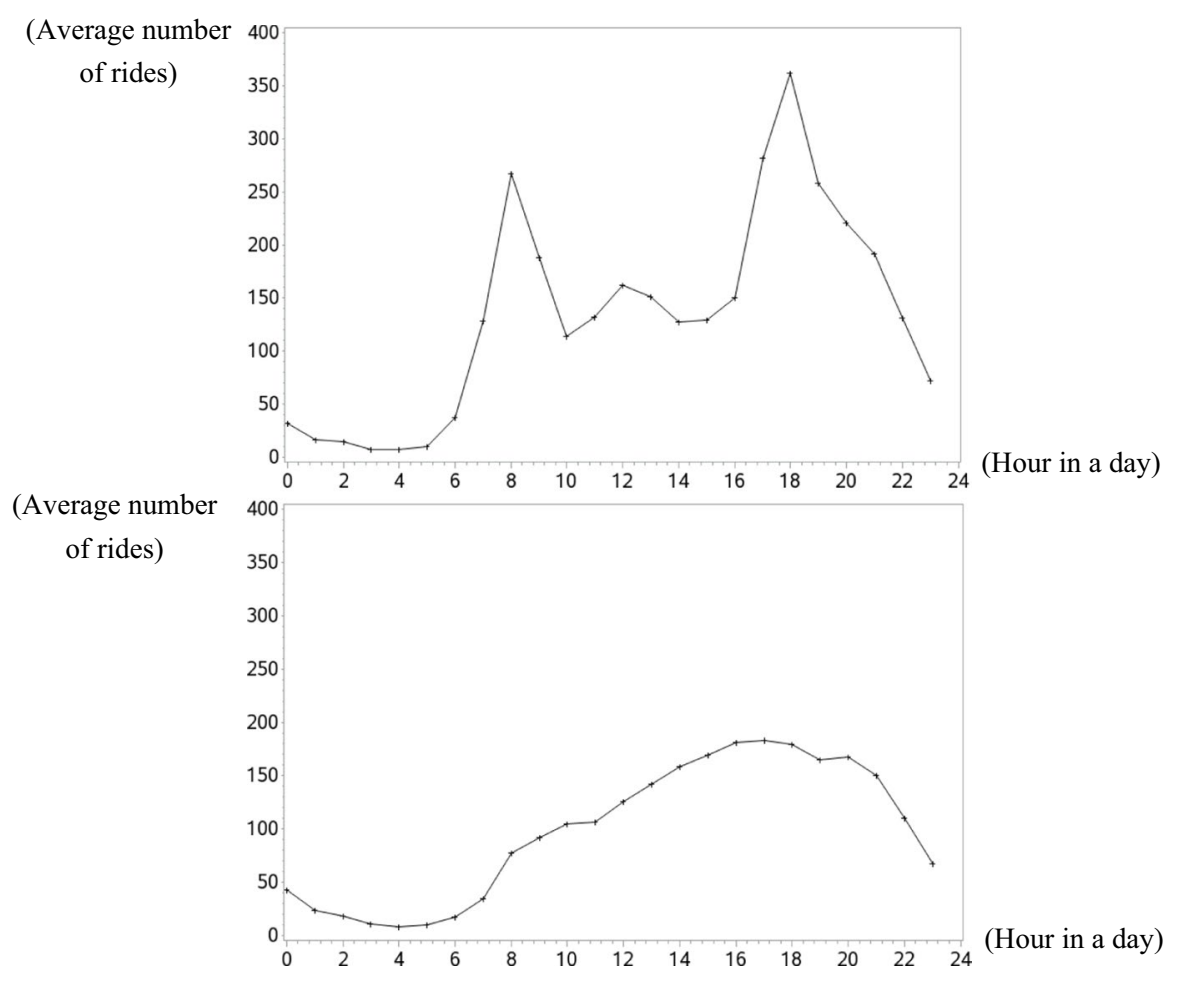

Fig. 3 Number of rides by hour. Note. The figures shows the average number of rides by hour. The $\mathrm{x}$-axis corresponds to time. For example, "8" refers to 8:00-8:59. The upper panel reports the patterns on weekdays, and the lower panel reports the patterns on weekends and holidays. The sample period is May to June, 2016

We use the riding records on 9 October 2017 to compute our key variable ( $\ln B i k e)$, which measures the steady-state intensity of shared bike usage by the residents of a given neighborhood. This date is an ordinary Monday, with a weather suitable for riding. On this day, there were 764,802 rides, with 136,262 individual bikes in operation. Each bike was ridden 5.6 times on average. This day was 18 month later than the launch of Meituan Bike. We assume that the riding patterns on this day is representative for the steady state. In the robustness check section, we will show that the spatial distribution of rides on this day is almost the same as that in 2019 or 2020 .

When calculating $\ln B i k e$, we use the rides during rush hours. On 9 October $2017,39 \%$ of the rides occurred during rush hours. These rides reflect the routine usage of shared bikes by commuters, who are perhaps the most stable and important user group of bike sharing companies. More specifically, we regard 7:30-9:30 as the morning rush hours. For each neighborhood, we count the number of rides that start from a point that is less than $200 \mathrm{~m}$ away from the neighborhood, and denote it as MornBike. Similarly, we regard 17:30-19:30 as the 
Table 1 Summary statistics of rush-hour rides

\begin{tabular}{llllll}
\hline & Mean & Std & P25 & P50 & P75 \\
\hline Distance (m) & 1200 & 1285 & 434 & 790 & 1474 \\
Time (min.) & 10 & 17 & 4 & 7 & 12 \\
StartToSub (m) & 581 & 541 & 222 & 484 & 738 \\
EndToSub (m) & 622 & 634 & 263 & 518 & 772 \\
\hline
\end{tabular}

Note. This table reports the summary statistics of the riding distance, riding time, the distance from the starting point to the nearest subway station (StartToSub), and the distance from the ending point to the nearest subway station (EndToSub). Here we only consider the rides that started during 7:30-9:30 and those that ended during 17:30-19:30 on 9 October 2017 . We report the mean, standard deviation, $25^{\text {th }}$ percentile, $50^{\text {th }}$ percentile, and $75^{\text {th }}$ percentile

evening rush hours. For each neighborhood, we count the number of rides that ends at a point that is less than $200 \mathrm{~m}$ away from the neighborhood, and denote it as EvenBike. Then $\ln B i k e$ is the natural logarithm of the sum of MornBike and EvenBike. In the robustness check section, we use $100 \mathrm{~m}$ and $300 \mathrm{~m}$ as alternative bandwidths when conducting robustness checks.

Table 1 reports the summary statistics of the rides during rush hours. For an average ride, the distance is $1.2 \mathrm{~km}$, and the duration is $10 \mathrm{~min}$; the distance from the starting (ending) point to the nearest subway station is $0.58 \mathrm{~km}(0.62 \mathrm{~km})$. Moreover, according to the (un-tabulated) results, about $20 \%(41 \%)$ of the rides either starts from a point that is less than $100 \mathrm{~m}(200 \mathrm{~m})$ away from a subway station, or ends at such a point. This suggests that a significant proportion of the riders use shared bikes as a complement to the subway network.

\section{House Transaction Data}

In our context, a "house" or "house unit" refers predominantly to an apartment unit. The house transaction data is from Lianjia, which is one of the largest real estate brokerages in Chinese cities (Li, Wei, Wu, and Tian, 2019). In 2018, 175,061 second-hand houses were transacted in Shanghai, according to Shanghai Bureau of Statistics. Among these houses, 30,963 were transacted through Lianjia, which translates to a market share of $18 \%$. The real estate brokerage industry of Shanghai is highly decentralized, and Lianjia is the largest brokerage in this city.

Our sample period is from 6 September 2011 to 13 July 2020. It straddles the launch of Meituan Bike, and also includes a period after the breakout of COVID-19. We have information of all the transactions made through Lianjia in Shanghai, but we need to limit our sample area to the region covered by the Meituan Bike dataset. This results in 79,963 house transactions in our sample. These houses are located in 4,415 neighborhoods from 120 zones. ${ }^{11}$ Figure 4 shows the location of the neighborhoods in which our sample houses are located.

\footnotetext{
${ }^{11}$ Lianjia divides Shanghai into 207 zones. Houses in the same zone have similar amenities and infrastructure quality.
} 


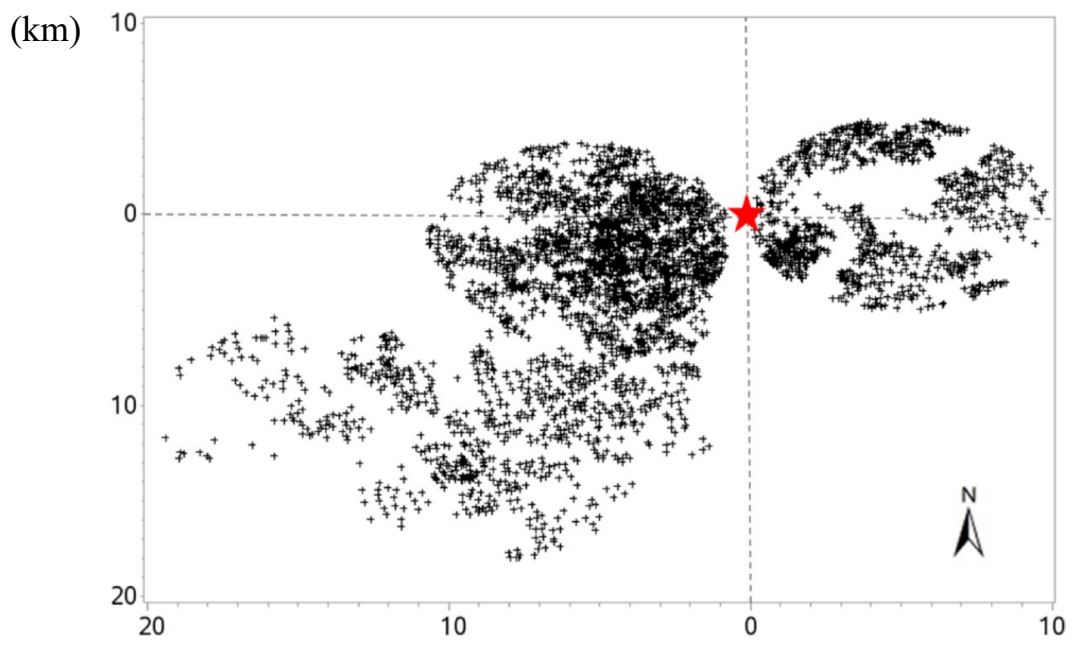

Fig. 4 Location of neighborhoods. Note. This figure shows the location of our sample neighborhoods relative to the city center (i.e. People's Square), which is the $(0,0)$ point and is marked by the red star. The $\mathrm{x}$-axis and the $\mathrm{y}$-axis shows the distance $(\mathrm{km})$ to the city center, respectively

The Lianjia dataset contains variables related to house characteristics, building characteristics, and neighborhood characteristics. The house characteristics include transaction price, transaction date, house size, the number of bedrooms, the number of parlors, house direction, interior decoration level, and the position in the building (underground/low/medium/high). The building characteristics include the total number of floors, building age, and building type (tower-type/slab-type/hybrid/bungalow). The neighborhood characteristics include neighborhood name, the management fee (per $\mathrm{m}^{2}$ per month) at the end of 2019 , the zone to which it belongs, and latitude and longitude. In our database, apartment buildings in the same neighborhood have the same longitude and latitude. Our key variable, $\ln B i k e$, is also a neighborhood-level variable.

Table 2 reports the summary statistics of the characteristics. An average house was transacted at 4,964,800 yuan (i.e., about 763,815 U.S. dollars), and the size is $90 \mathrm{~m}^{2}$. It has 2 bedrooms, 1 parlor, and a medium position in the building. The building has 14 levels and was built 19 years before the transaction. The building is located in a neighborhood with a management fee of $1.88 \mathrm{yuan} / \mathrm{m}^{2} / \mathrm{month}$ at the end of 2019 .

\section{POI Information}

We collect point-of-interest (POI) information by using the API service of Baidu Map and AMap, both of which are Chinese counterparts of Google Maps. With the API service, one can input a keyword, and the server of the maps will return the names and locations of the POIs related to that keyword. For different keywords, the efficiency of the two maps is different. Given a keyword, we try both maps, and retain the more efficient set of results. We obtain the locations of subway stations 
Table 2 Summary statistics of house characteristics

\begin{tabular}{lrlr}
\hline & Mean & Median & \multicolumn{1}{c}{ Std } \\
\hline Price & 496.48 & 382 & 386.76 \\
Size & 90.29 & 76.97 & 53.09 \\
Room & 2.06 & 2 & 0.87 \\
Parlor & 1.38 & 1 & 0.68 \\
Position & 3.06 & 3 & 0.80 \\
TotFloor & 14.15 & 7 & 10.45 \\
Age & 19.01 & 18 & 9.50 \\
ManaFee & 1.88 & 1.29 & 2.75 \\
lnBike & 4.78 & 4.95 & 1.07 \\
DSub & 0.80 & 0.63 & 0.65 \\
DCenter & 8.42 & 7.34 & 4.67 \\
\hline
\end{tabular}

Note. Price is transaction price (in 10 thousand yuan). Size is the house size $\left(\mathrm{m}^{2}\right)$. Room and Parlor are the numbers of bedrooms and parlors, respectively. Position is an integer that equals 1, 2, 3, or 4 if the house is located at underground level, low level, medium level, or high level in the building, respectively. TotFloor is the number of floors in the building. Age is the building age at the time of transaction. ManagFee is the property management fee (yuan $/ \mathrm{m}^{2} / \mathrm{month}$ ) of the neighborhood. For some neighborhoods, the management fee included in the dataset is a range instead of a single number; in this case, we calculate the fee as the average of the minimum and the maximum. The variable $\ln$ Bike is a measure of the intensity of shared bike usage in the steady state. DSub and DCenter is the distance $(\mathrm{km})$ from a house to the nearest subway station and the city center (i.e. People's Square), respectively

from AMap, and obtain the locations of City Management Team (CMT) offices from Baidu Map. Figure 5 show the distribution of CMT offices in Shanghai.

\section{Empirical Results}

We first examine the impact of bike sharing on house prices in normal time. Then we investigate how COVID-19 has changed the house price effects of bike sharing.

\section{Bike Sharing and House Prices in Normal Time}

We run the baseline regression (1) for the sample period 2011-2019, which is before the breakout of COVID-19. Table 3 Column (1) shows the regression results. The coefficient of $\ln B i k e$ is significantly negative, indicating that a high density of Meituan Bike usage is associated with relatively low house prices in the pre-launch period. This is understandable, because high-income households usually have private cars and do not rely on shared bikes or subways. But meanwhile, 


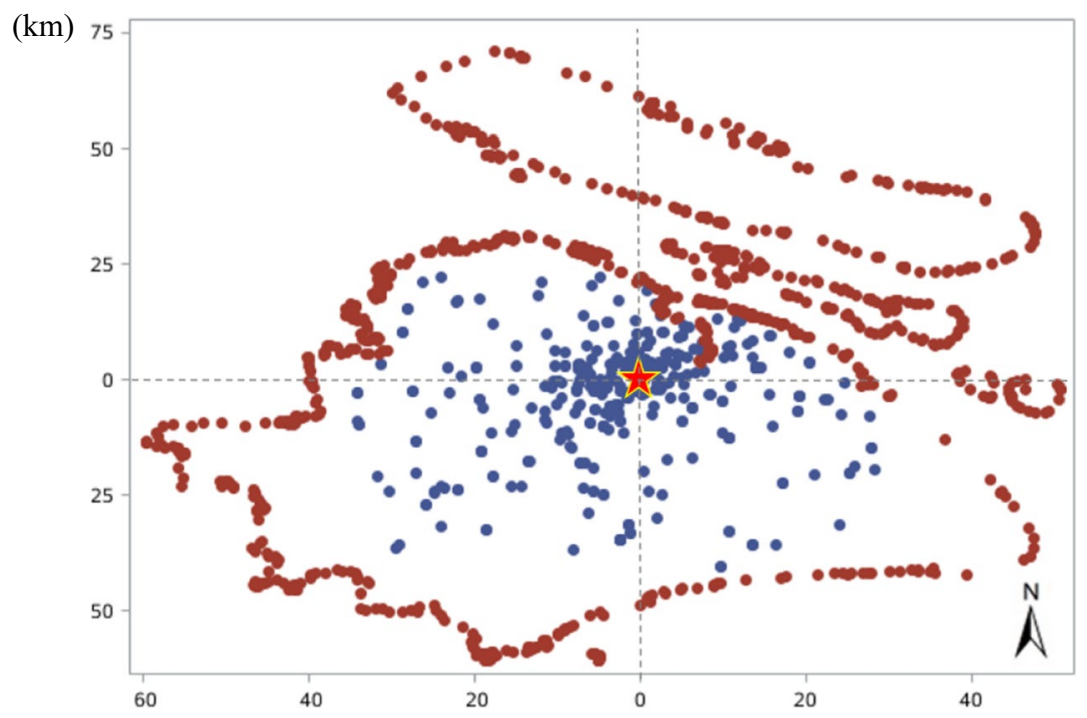

Fig. 5 The distribution of CMT offices. Note. This blue dots show the location of city management team (CMT) offices relative to the city center, which is the $(0,0)$ point and marked by the red star. The red dots indicate the boundary of Shanghai. The $\mathrm{x}$-axis and the $\mathrm{y}$-axis shows the distance (km) to the city center, respectively. The POI data was obtained from Baidu Map in January 2020

this introduces the endogeneity concern related to $\ln B i k e$; we will address this concern in the robustness check section. The coefficient of $\ln B i k e^{*}$ Post is negative, but is insignificant. This suggests a modestly negative external effect of bike sharing, which provides limited support for $\mathrm{H} 2$.

The coefficient of $D S u b$ is significantly negative. Since $D S u b$ measures the distance to the nearest subway station, a negative coefficient indicates a positive subway premium. Moreover, the coefficient of $D S u b^{*}$ Post is insignificant, while the coefficient of $\ln B i k e^{*} D S u b^{*}$ Post is significantly positive. That is, during the post-launch period, the subway premium has not changed much in areas with little shared bike usage. In contrast, the premium has been significantly reduced in areas where shared bikes are intensively used. As $\ln B i k e$ increases from the $25^{\text {th }}$ percentile $(4.33)$ to the $75^{\text {th }}$ percentile $(5.47)$, the premium reduction relative to its pre-launch level increases from $24.8 \%$ (i.e., $4.33 * 0.0073 / 0.1277$ ) to $31.3 \%$ (i.e., $5.47 * 0.0073 / 0.1277$ ). These results are consistent with $\mathbf{H 1}$, providing evidence for the positive value of bike sharing as a complement to the subway network.

The (unreported) coefficients of the controlling variables are also consistent with our expectations. For example, house price increases with house size and the numbers of bedrooms and parlors, and decreases with the distance to the city center. For high-rise buildings, house price is higher for higher levels; for low-rise buildings, which typically do not have elevators, house price is lower for higher levels. 
Table 3 Shared bike usage intensity and house prices

\begin{tabular}{|c|c|c|c|}
\hline & \multirow{2}{*}{$\begin{array}{l}\text { Pre-COVID } \\
\text { (1) }\end{array}$} & \multicolumn{2}{|l|}{ Full sample } \\
\hline & & $\begin{array}{l}\text { (2) PostCOVID: } 1 \text { Jan } \\
2020\end{array}$ & $\begin{array}{l}\text { (3) Post- } \\
\text { COVID: } 23 \\
\text { Jan } 2020\end{array}$ \\
\hline \multirow[t]{2}{*}{ lnBike } & $-0.0462^{* * *}$ & $-0.0453^{* * *}$ & $-0.0453^{* * *}$ \\
\hline & $(0.0091)$ & $(0.0091)$ & $(0.0091)$ \\
\hline \multirow[t]{2}{*}{ lnBike*DSub } & 0.0082 & 0.0084 & 0.0084 \\
\hline & $(0.0056)$ & $(0.0056)$ & $(0.0056)$ \\
\hline \multirow[t]{2}{*}{ DSub } & $-0.1277^{* * *}$ & $-0.1291^{* * *}$ & $-0.1292^{* * *}$ \\
\hline & $(0.0218)$ & $(0.0217)$ & $(0.0217)$ \\
\hline \multirow[t]{2}{*}{ InBike*Post } & -0.0061 & -0.0059 & -0.0061 \\
\hline & $(0.0050)$ & $(0.0050)$ & $(0.0049)$ \\
\hline \multirow[t]{2}{*}{ lnBike*DSub*Post } & $0.0073^{* *}$ & $0.0072^{* *}$ & $0.0075^{* *}$ \\
\hline & $(0.0029)$ & $(0.0029)$ & $(0.0029)$ \\
\hline \multirow[t]{2}{*}{ DSub*Post } & -0.0011 & -0.0013 & -0.0020 \\
\hline & $(0.0105)$ & $(0.0105)$ & $(0.0105)$ \\
\hline \multirow[t]{2}{*}{ InBike*PostCOVID } & & $-0.0090^{*}$ & -0.0082 \\
\hline & & $(0.0053)$ & $(0.0056)$ \\
\hline \multirow[t]{2}{*}{ lnBike*DSub*PostCOVID } & & $0.0085^{* *}$ & $0.0070^{*}$ \\
\hline & & $(0.0036)$ & $(0.0037)$ \\
\hline \multirow[t]{2}{*}{ DSub*PostCOVID } & & $-0.0317^{* *}$ & $-0.0283^{*}$ \\
\hline & & $(0.0144)$ & $(0.0149)$ \\
\hline Controls & $\mathrm{Y}$ & $\mathrm{Y}$ & $\mathrm{Y}$ \\
\hline Sample period & 2011-2019 & 2011-2020 & 2011-2020 \\
\hline Obs & 67,339 & 74,961 & 74,961 \\
\hline $\mathbf{R}^{2}$ & $81.43 \%$ & $81.84 \%$ & $81.83 \%$ \\
\hline
\end{tabular}

Note. The dependent variable is the natural logarithm of transaction price. The definitions of independent variables are summarized in Appendix A. Numbers in parentheses are standard errors, which are clustered by neighborhood. The significant levels of $10 \%, 5 \%$, and $1 \%$ are marked with ${ }^{*},{ }^{* *}$, and ${ }^{* * *}$, respectively

\section{Further Investigation on the Negative External Effect}

In Table 3 Column (1), the coefficient of $\ln B i k e^{*}$ Post is insignificantly negative, suggesting a slightly negative effect of bike sharing on house prices. However, this result may mask the heterogeneity across neighborhoods. For example, luxury neighborhoods usually have high-quality property management service. It may be better to exclude such neighborhoods when investigating the negative external effect, because the service usually includes re-arranging misplaced shared bikes in and around the neighborhood.

Figure 6 displays the distribution of property management fees of our sample houses at the end of 2019. Houses in the same neighborhood have the same property management fee (per square meter per month). As can be seen from the figure, there is a jump at 5 yuan $/ \mathrm{m}^{2} /$ month. This is also the $95^{\text {th }}$ percentile. We classify houses with a property management fee no more than $5 \mathrm{yuan} / \mathrm{m}^{2} / \mathrm{month}$ 
Fig. 6 Distribution of property management fee. Note. This figure displays the distribution of property management fee (yuan) of our sample houses in 2019

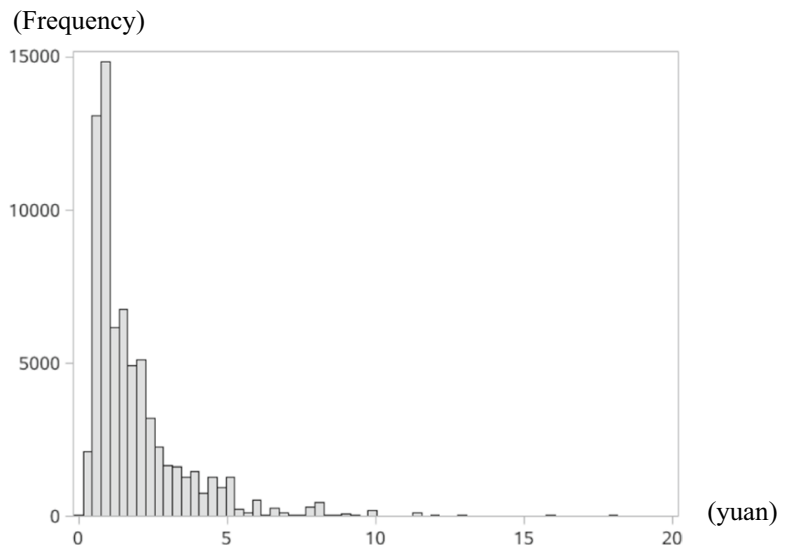

into the "Ordinary" group, and classify those with higher fees into the "Luxury" group. Then we repeat the baseline regression (1) separately for the two groups.

Table 4 Column (1) reports the results for the Ordinary group. The coefficient of $\ln B i k e^{*}$ Post is significantly negative. That is, for neighborhoods near subway

Table 4 Subsample analysis

\begin{tabular}{|c|c|c|c|c|}
\hline & \multicolumn{2}{|c|}{ By management fee } & \multicolumn{2}{|c|}{ By distance to CMT offices } \\
\hline & (1)Ordinary & (2)Luxury & (3)Near & (4)Far \\
\hline \multirow[t]{2}{*}{ InBike } & $-0.0256^{* * *}$ & $-0.1764^{* * *}$ & $-0.0713^{* * *}$ & $-0.0302^{* *}$ \\
\hline & $(0.0096)$ & $(0.0390)$ & $(0.0161)$ & $(0.0125)$ \\
\hline \multirow[t]{2}{*}{ InBike*DSub } & 0.0046 & 0.0476 & $0.0504^{* *}$ & 0.0085 \\
\hline & $(0.0053)$ & $(0.0417)$ & $(0.021)$ & $(0.0057)$ \\
\hline \multirow[t]{2}{*}{ DSub } & $-0.1095^{* * * *}$ & $-0.2230^{*}$ & $-0.3565^{* * *}$ & $-0.1071^{\text {*** }}$ \\
\hline & $(0.0197)$ & $(0.1296)$ & $(0.1053)$ & $(0.0222)$ \\
\hline \multirow[t]{2}{*}{ InBike*Post } & $-0.0100^{* *}$ & 0.0188 & 0.0009 & -0.0103 \\
\hline & $(0.0048)$ & $(0.0221)$ & $(0.0081)$ & $(0.0067)$ \\
\hline \multirow[t]{2}{*}{ InBike*DSub*Post } & $0.0086^{* * *}$ & 0.0060 & -0.0056 & $0.0090^{* * *}$ \\
\hline & $(0.0029)$ & $(0.0164)$ & $(0.0127)$ & $(0.0035)$ \\
\hline \multirow[t]{2}{*}{ DSub*Post } & -0.0070 & -0.0426 & 0.0632 & -0.0130 \\
\hline & $(0.0105)$ & $(0.0425)$ & $(0.0561)$ & $(0.0122)$ \\
\hline Controls & $\mathrm{Y}$ & $\mathrm{Y}$ & $\mathrm{Y}$ & $\mathrm{Y}$ \\
\hline Obs & 64,863 & 2476 & 33,422 & 33,917 \\
\hline $\mathbf{R}^{2}$ & $81.16 \%$ & $86.84 \%$ & $81.81 \%$ & $82.48 \%$ \\
\hline
\end{tabular}

Note. The dependent variable is the natural logarithm of transaction prices. Columns (1) focus on ordinary neighborhoods, which have a property management fee of no more than $5 \mathrm{yuan} / \mathrm{m}^{2} / \mathrm{month}$ (i.e. the $95^{\text {th }}$ percentile). Column (2) focus on luxury neighborhoods, which have a fee higher than $5 \mathrm{yuan} / \mathrm{m}^{2} /$ month. We run regression (1) separately for each group. In columns (3) and (4), we classify houses into two groups according to the distance to the nearest CMT office. The breakpoint is the median distance. Numbers in parentheses are standard errors, which are clustered by neighborhood. The significant levels of $10 \%, 5 \%$, and $1 \%$ are marked with ${ }^{*}{ }^{* *}$, and ${ }^{* * *}$, respectively 
stations, the relationship between $\ln B i k e$ and house price has become more negative after the launch of Meituan Bike. This is consistent with H2a. As the distance to subway stations increases, the positive value of bike sharing becomes more evident, and the break-even distance is $1.2 \mathrm{~km}$ (i.e. 0.0100/0.0086). This is consistent with the intuition that people may prefer walking to riding a shared bike if the distance is not very long.

Our findings are robust if we replace $D S u b$ with the dummy Far, which equals 1 if $D S u b$ is above the break-even distance $1.2 \mathrm{~km}$, and 0 otherwise. In this specification, the coefficient of $\ln B i k e^{*}$ Post is $-0.0073(\mathrm{p}=0.09)$, and the coefficient of $\ln B i k e^{*} F_{a r}^{*}$ Post is 0.0255 ( $\mathrm{p}<0.01$ ), according to the (un-tabulated) results.

Table 4 Column (2) reports the results for the Luxury group. The coefficient of $\ln B i k e^{*}$ Post is positive and insignificant. This suggests that the high-quality property management service effectively eliminates the negative external effect of bike sharing. ${ }^{12}$

Then we classify houses into the "Near" group and the "Far" group according to their distance to the nearest CMT office. We use the median distance $(0.6 \mathrm{~km})$ as the breakpoint in the classification. Then we repeat regression (1) separately for the two groups. The results are displayed in Table 4 Column (3) to (4). For the "Near" group, the coefficient of $\ln B i k e^{*}$ Post is positive and insignificant. This indicates that in places well-covered by CMTs, the negative external effect of bike sharing is eliminated. For the "Far" group, the coefficient of $\ln$ Bike* Post is negative and marginally significant $(\mathrm{p}=0.12)$. Hence, in places poorly covered by CMTs, the negative external effect is stronger. These results are also consistent with H2a.

Another interesting finding from Table 4 is that, while high-quality property management service and CMTs effectively eliminate the negative external effect of bike sharing, they also eliminate the positive value. The coefficient of $\ln B i k e^{*} D S u b^{*}$ Post is insignificant for the "Luxury" group and the "Near" group. This is unsurprising, because when shared bikes are "rearranged" by the staff of property management companies or CMTs, they are basically moved to a place with fewer people (and thus fewer potential users).

The above results indicate that better approaches are needed to eliminate the negative external effect without reducing the positive value. One possible approach is creating a list of "correct" parking locations. Hellobike, which is the competitor of Meituan Bike, is trying to do this by collaborating with the government of Songjiang District of Shanghai. They will make a list of "correct" parking spaces. A user cannot check out in the system unless he/she parks the bike in a "correct" location. ${ }^{13}$ As long as the "correct" list is properly designed, it is possible to eliminate the negative external effect caused by misplacement, while still maintaining the convenience of shared bikes to most users.

\footnotetext{
${ }^{12}$ An alternative way of doing the analysis is running a full-sample regression that includes Fee, which is the property management fee. This would result in additional interaction terms, such as $\ln \mathrm{Bike}^{*} \mathrm{DSub} \boldsymbol{P}^{\mathrm{Pos}} \boldsymbol{F}^{*} \mathrm{Fee}$ and more triple-way interaction terms. According to the results, the coefficient of $\ln B i k e^{*}$ Post is significantly negative, and the coefficient of $\ln B i k e^{*}$ Post ${ }^{*}$ Fee is positive $(\mathrm{p}=0.27)$. Furthermore, the coefficient of $\ln B i k e^{*} D S u b^{*}$ Post is significantly positive, and the coefficient of $\ln \mathrm{Bike}^{*} \mathrm{DSub} \mathrm{Post}^{*} \mathrm{Fee}$ is significantly negative. In general, these results are consistent with our findings based on subsample analysis, and are available upon request.

${ }^{13}$ Please see more details about this project at Sohu (in Chinese), which is a famous daily news website in China: https://www.sohu.com/a/408692624_120244154
} 


\section{The Impact of a Pandemic on the Relationship Between Bike Sharing and House Prices}

Now we investigate whether the effects of bike sharing has been more or less capitalized into house prices after the breakout of COVID-19. We extend our sample period to 13 July 2020, and add the interaction terms involving the dummy PostCOVID into the right-hand side of the baseline regression.

The results are reported in Table 3 Column (2). The coefficient of $\ln B i k e^{*}$ PostCOVID is significantly negative, and the coefficient of $\ln B i k e^{*} D S u b^{*}$ PostCOVID is significantly positive. That is, both the positive value and negative external effect of bike sharing has been more heavily capitalized into house prices since the breakout of the pandemic. These results support $\mathbf{H 3}$.

In Table 3 Column (3), we use a more conservative definition for the post-pandemic period. More specifically, we redefine PostCOVID as a dummy that equals 1 for the dates after 23 January 2020, and 0 for earlier dates. The breakpoint is the day when Wuhan, China was locked down. The results are similar to those in Columns (2).

The above finding is consistent with the fact that both the extensity and intensity of shared bike usage has increased during the pandemic. From 9 October 2019 to 9 June 2020 , the number of rush-hour users increased by $2 \%$, and the number of rush-hour rides increased by $4 \%$; this is likely due to the advantage of shared bikes in allowing for social distance. The increased user base makes people more confident about the long survival of the bike sharing industry, which strengthens its house price effects.

Some may be surprised at the increase in the positive value of bike sharing as a complement to subways, because social distancing is supposed to discourage people from taking a subway. However, it is worth noticing that the drop of subway passenger volume was mainly temporary. As displayed in Fig. 7, the passenger volume of Shanghai subways rebounded quickly after February 2020. Till July 2020, the

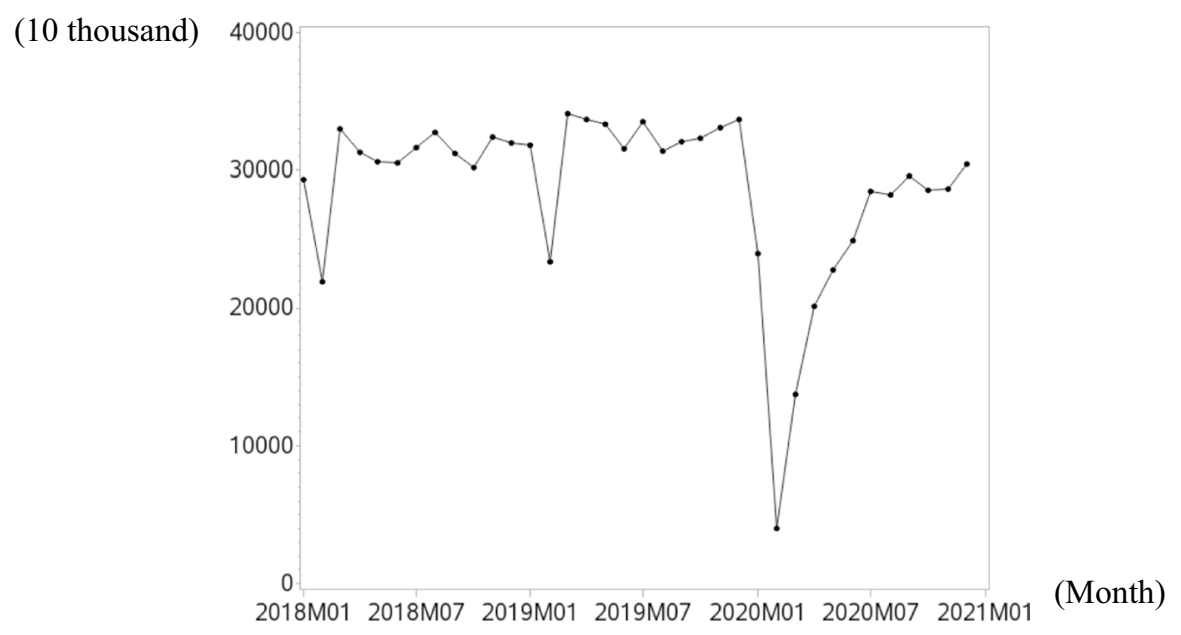

Fig. 7 Passenger volume of Shanghai subways. Notes. This figure shows the passenger volume (in 10 thousand) of the subway network in Shanghai. The sample period is from January 2018 to December 2020 
volume already reached $85 \%$ of the volume in July 2019 . Hence, the long-term value of bike sharing as a complement to the subway network remains. ${ }^{14}$

\section{Robustness Checks}

In this section, we alleviate the endogeneity concern. We also show that our findings are robust if we use alternative dates and alternative bandwidths when computing our key variable, if we use alternative empirical models, and if we control for the effects of neighborhood population. We further show that our findings are robust if we allow for a transition period, or take into account the expansion of the subway network during our sample period.

\section{The Endogeneity Concern and Placebo Tests}

Some may be concerned about the endogeneity problem associated with our key variable lnBike. In the baseline regression (1), its coefficient is significantly negative, suggesting that cheap houses are associated with higher $\ln B i k e$. If the price of proximity to subway stations has a more negative time trend for cheap houses than for expensive houses, then the positive coefficient of $\ln B i k e^{*} D S u b^{*}$ Post cannot be attributed to the positive value of bike sharing. However, this is unlikely, and the reasoning is as followed. From 2011 to 2019, the annual passenger volume of Shanghai subways increased by as much as $86 \%$ (from 2.1 billion to 3.9 billion), according to National Bureau of Statistics (NBS) of China. During this period, the route length of the subway network increased by $55 \%$ (from 454 to $705 \mathrm{~km}$ ), according to Shanghai Bureau of Statistics. Since the newly opened stations are mostly located in suburb and have smaller passenger volume than the old stations in the downtown, the growth of route length cannot fully explain the large growth in the total passenger volume. Neither can population growth explain it; the total population in Shanghai increased by merely 3\% from 2011 to 2019, according to NBS. Hence, we infer that in general, people's tendency of taking a subway has increased. The increase in the tendency of taking a subway is more likely to be driven by low-income households than by high-income households, because low-income households have lower car-ownership rate and are more reliant on subways. Assuming that low-income households live in relatively cheap houses, the value of proximity to subway stations should increase more for cheap houses than for expensive houses.

Another related concern is that, if the price of cheap houses has a more negative time trend than that of expensive houses, then the negative coefficient of $\ln B i k e^{*}$ Post

\footnotetext{
14 The two negative spikes in the February of 2018 and 2019 were due to the Chinese New Year, when people return to their hometown and enjoy family reunion.
} 
cannot be attributed to the negative external effect of bike sharing. But this is also unlikely. For each month, we compute the $25^{\text {th }}$ percentile and the $75^{\text {th }}$ percentile of the transaction prices, and then calculate the ratio of the former to the latter. From March 2016 (the month before the launch) to July 2020, the ratio gradually increased from 0.36 to 0.50 . Thus, the price of cheap houses actually has a positive time trend relative to that of expensive houses.

To further mitigate the time trend concern, we conduct placebo tests, using ordinary neighborhoods and the normal period 2011-2019. More specifically, we use different cutoff points when defining Post. We start from January 2013 and end at December 2018. That is, we first redefine Post as a dummy that equals 1 for transactions in January 2013 or later, and 0 for earlier transactions. With the new Post, we repeat the baseline regression (1) and record the coefficients of $\ln B i k e^{*} D S u b^{*}$ Post, which reflects the positive value of bike sharing. Then we repeat the above procedures by replacing January 2013 with February 2013 and so on, until December 2018. The results are displayed by Fig. 8a. The coefficient is significant in the months around the true launch time, and mostly insignificant in other periods.

Then we do the same analysis for the coefficient of $\ln B i k e^{*}$ Post, which reflects the negative external effect of bike sharing. The results are shown in Fig. 8b. The coefficient of $\ln B i k e^{*}$ Post is significant when the cutoff point is around the true launch month, and is mostly insignificant in other periods.

Therefore, a randomly defined post-launch period is unlikely to generate significant results. Our major findings only hold when the cutoff point belongs to a small window around the true launch time. Stories based on time trend cannot explain the coincidence between this small window and the true launch time. Some may be concerned that the small window coincided with a housing boom in Shanghai, and the pricing of house characteristics during a boom may be different from that in normal time. But if so, we should observe significant coefficients when the cutoff line is set either before the boom or after the boom, not in the middle of the boom.

\section{Alternative Dates for Calculating InBike}

So far, we have used the riding records on 9 October 2017 to calculate $\ln$ Bike. We assume that this date is representative for the steady state. To test the validity of this assumption, we use alternative dates to compute $\ln B i k e$. In particular, we recalculate it with the riding records on 9 October 2019 and 9 June 2020, separately. ${ }^{15}$ The former day is representative for the usage intensity immediately before the breakout of COVID-19, and the latter day is representative for the intensity in the post-pandemic period. ${ }^{16}$

We find that the correlation between the original value of $\ln B i k e$ and the value based on 9 October 2019 (9 June 2020, respectively) is 0.88 (0.90,

\footnotetext{
159 October 2019 was a Wednesday, with a temperature ranging from $19^{\circ} \mathrm{C}$ to $24^{\circ} \mathrm{C}$ (i.e., $66^{\circ} \mathrm{F}$ to $75^{\circ} \mathrm{F}$ ). 9 June 2020 was a Tuesday, with a temperature ranging from $24^{\circ} \mathrm{C}$ to $28^{\circ} \mathrm{C}$ (i.e., $75^{\circ} \mathrm{F}$ to $82^{\circ} \mathrm{F}$ ). Both days were cloudy and suitable for riding.

16 In June 2020, the pandemic was almost ended. On 9 June 2020, there were only 3 new cases in China. The data source is Wind database.
} 
Fig. 8 a Placebo tests for the positive value. b Placebo tests for the negative external effect. Note. We redefine Post using different cutoff points between January 2013 and December 2018, and rerun the baseline regression (1). We consider ordinary neighborhoods and use the sample period 2011-2019. Standard errors are clustered by neighborhood. This table displays the coefficients of $\ln$ Bike*Post corresponding to these cutoff points. The solid line shows the estimated coefficient. The shadow area shows the $90 \%$ confidence interval of the estimates. The vertical line marks April 2016, which is the true launch month

respectively). That is, the distribution of the usage intensity is quite stable during the period 2017-2020, and even COVID-19 did not change it. Then we rerun regression (1) by replacing the original $\ln B i k e$ with the new values; we consider ordinary neighborhoods and use the sample period 2011-2020. The results are displayed in Table 5. The coefficient of $\ln B i k e^{*}$ Post is significantly negative, no matter whether we use the records on 9 October 2019 or 9 June 2020 when calculating $\ln B i k e$. This is consistent with H2. In addition, the coefficient of $\ln B i k e^{*} D S u b^{*}$ Post is significantly positive in Column (1), and marginally significant in Column (2) $(\mathrm{p}=0.105)$. This is consistent with H1.

\section{Alternative Bandwidth}

When calculating $\ln$ Bike for a neighborhood, we consider the rush-hour rides that start or end at a place that is less than $200 \mathrm{~m}$ from the central point of the neighborhood. Now we examine whether our findings are robust to alternative bandwidths. Instead of $200 \mathrm{~m}$, we consider $100 \mathrm{~m}$ and $300 \mathrm{~m}$. Then we recalculate $\ln B i k e$ and rerun regression (1). We consider ordinary neighborhoods and use a sample period from 2011 to 2020 .

Table 6 Column (1) adopts a bandwidth of $100 \mathrm{~m}$. The coefficient of $\ln$ Bike*Post remains negative, though insignificant. Meanwhile, the coefficient of $\ln B i k e^{*} D S u b^{*}$ Post is significantly positive. Column (2) adopts a bandwidth of $300 \mathrm{~m}$. The coefficient of $\ln$ Bike*Post is significantly negative, and the coefficient of $\ln B i k e^{*} D S u b^{*}$ Post is significantly positive. Therefore, our findings are robust to alternative bandwidths. The hypotheses $\mathrm{H} 1$ and $\mathrm{H} 2$ are supported.

\section{Allowing for a Transition Period}

This sub-section examines whether our findings are robust if we exclude a transition period. Meituan Bike was launched in 22 April 2016. Defining this day as day $t$, we drop the sub-period $[t-90, t+90]$ from our sample period 2011-2019, and repeat the baseline regression (1). We consider ordinary neighborhoods. According to the (un-tabulated) results, the coefficient of $\ln$ Bike*Post is $-0.0124(\mathrm{p}=0.06)$, and the coefficient of $\ln B i k e^{*} D S u b^{*}$ Post is $0.0092(\mathrm{p}=0.01)$. They are consistent with H1 and H2. 

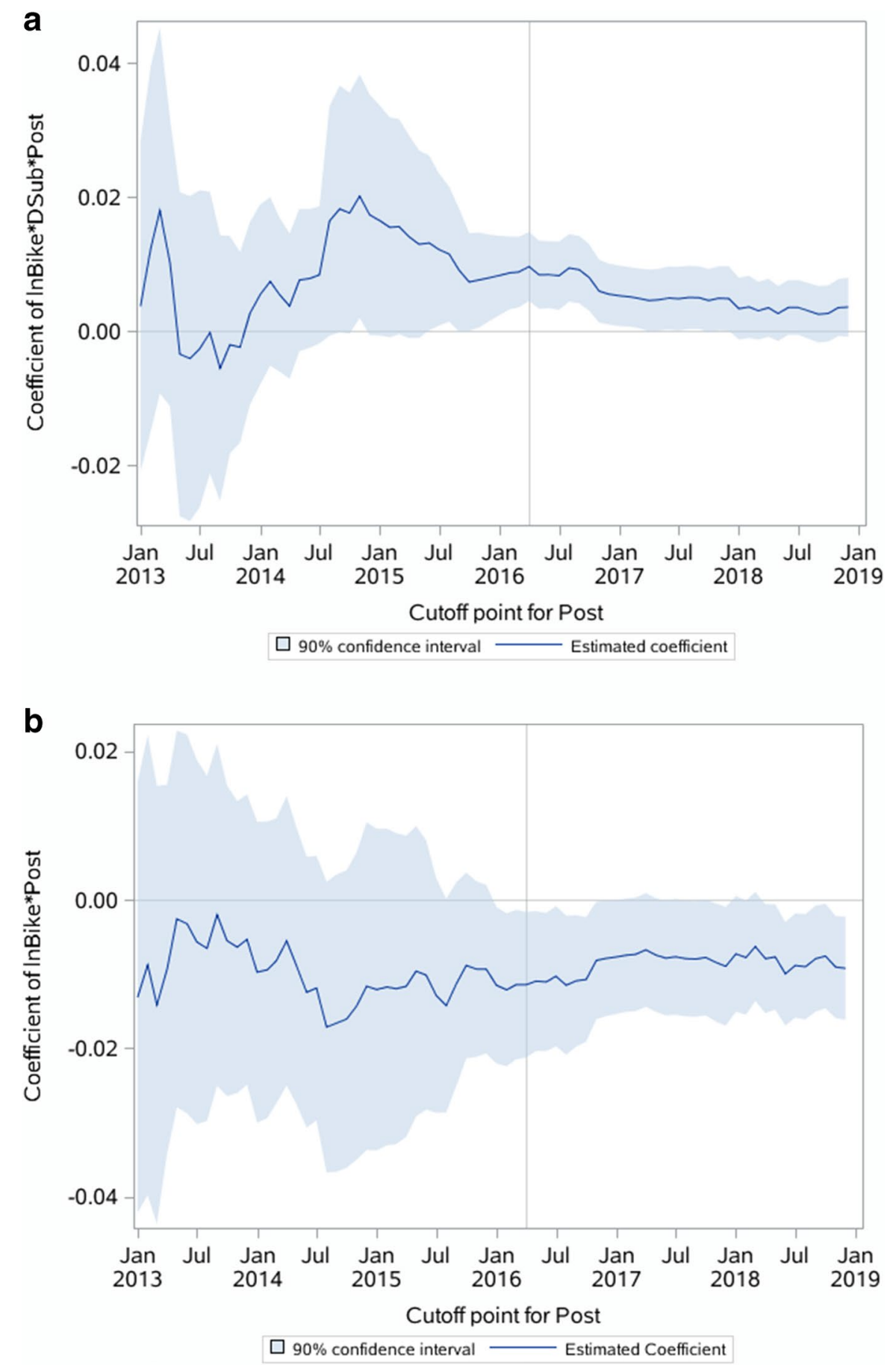
Table 5 Alternative dates of riding records
(1)9 Oct 2019

(2)9 June 2020

\begin{tabular}{lll}
\hline InBike & $-0.0239^{* * *}$ & $-0.0204^{* *}$ \\
& $(0.0088)$ & $(0.0079)$ \\
InBike*DSub & $0.0092^{*}$ & $0.0108^{* *}$ \\
& $(0.005)$ & $(0.0043)$ \\
DSub & $-0.1117^{* * *}$ & $-0.1121^{* * *}$ \\
& $(0.0176)$ & $(0.0223)$ \\
InBike*Post & $-0.0104^{* * *}$ & $-0.0101^{* * *}$ \\
& $(0.0039)$ & $(0.0035)$ \\
InBike*DSub*Post & $0.0064^{* *}$ & 0.0044 \\
& $(0.0029)$ & $(0.0027)$ \\
DSub*Post & 0.0030 & 0.0061 \\
& $(0.0081)$ & $(0.0080)$ \\
Controls & $\mathrm{Y}$ & $\mathrm{Y}$ \\
Obs & 72,339 & 72,339 \\
$\mathbf{R}^{2}$ & $81.59 \%$ & $81.59 \%$ \\
\hline
\end{tabular}

Note. The dependent variable is the natural logarithm of transaction prices. In Column (1), we replace the original $\ln$ Bike (which is based on the riding records on 9 October 2017) with a value based on the records on 9 October 2019. In Column (2), we replace it with a value based on the records on 9 June 2020. The sample period is 2011 to 2020 in both columns. We consider ordinary neighborhoods. Numbers in parentheses are standard errors, which are clustered by neighborhood. The significant levels of $10 \%, 5 \%$, and $1 \%$ are marked with ${ }^{*},{ }^{* *}$, and ${ }^{* * * *}$, respectively

\section{Controlling for Neighborhood Population}

Some may be concerned that both population and $\ln B i k e$ differ between neighborhoods, and the effects of $\ln B i k e$ that we have observed may be spuriously caused by its positive correlation with neighborhood population. To mitigate this concern, we directly control for the effects of neighborhood population. For each neighborhood, we know the number of house units in it. We scale this number by 1,000 and denoted it as HouseUnit, which is a neighborhood-level variable. We add four variables into the right-hand side of the baseline regression: HouseUnit, HouseUnit*DSub, HouseUnit*Post, and HouseUnit*DSub*Post. With these four additional variables, we repeat the baseline regression, using houses in ordinary neighborhoods and the sample period of 2011-2019.

According to the (un-tabulated) results, the coefficient of $\ln B i k e^{*}$ Post is $-0.0071(\mathrm{p}=0.12)$, and the coefficient of $\ln B i k e^{*} D$ Sub Post is $0.0067(\mathrm{p}=0.03)$. Thus, in general, our major results hold even after controlling for neighborhood population. In addition, the coefficients of HouseUnit and HouseUnit*DSub*Post are insignificant; the coefficients of HouseUnit*DSub and HouseUnit*Post are $-0.0051(\mathrm{p}=0.05)$ and $0.0042(\mathrm{p}=0.09)$, respectively. The significantly positive 
Table 6 Alternative bandwidth

\begin{tabular}{lll}
\hline & $(1) \mathbf{1 0 0} \mathbf{~ m}$ & $(2) \mathbf{3 0 0} \mathbf{~ m}$ \\
\hline InBike & $-0.0302^{* * * *}$ & $-0.0412^{* * *}$ \\
InBike*DSub & $(0.0066)$ & $(0.0124)$ \\
& 0.0017 & 0.0065 \\
DSub & $(0.0080)$ & $(0.0061)$ \\
& $-0.0984^{* * *}$ & $-0.1326^{* * *}$ \\
InBike*Post & $(0.0138)$ & $(0.0271)$ \\
& -0.0031 & $-0.0096^{* *}$ \\
InBike*DSub*Post & $(0.0040)$ & $(0.0047)$ \\
DSub*Post & $0.0107^{* * *}$ & $0.0124^{* * *}$ \\
& $(0.0034)$ & $(0.0031)$ \\
Controls & 0.0119 & $-0.0298^{* *}$ \\
Obs & $(0.0072)$ & $(0.0136)$ \\
R $^{2}$ & $\mathrm{Y}$ & $\mathrm{Y}$ \\
\hline
\end{tabular}

Note. The dependent variable is the natural logarithm of transaction prices. In Columns (1), we use a 100-m bandwidth when calculating $\ln$ Bike. In Columns (2), we use a 300-m bandwidth. The controlling variables are the same as in regression (1). The sample period is 2011 to 2020 . We consider ordinary neighborhoods. Numbers in parentheses are standard errors, which are clustered by neighborhood. The significant levels of $10 \%, 5 \%$, and $1 \%$ are marked with ${ }^{*}$, **, and ${ }^{* * *}$, respectively

coefficient of HouseUnit*Post indicates that the introduction of bike sharing is associated with a more positive house price change for larger neighborhoods. This is consistent with the intuition that, in Chinese cities, very large neighborhoods usually consist of old apartment buildings without elevators. They attract households with relatively low income, who are more likely to use shared bikes than richer households.

\section{Alternative Empirical Models}

Now we show that our major findings are robust to alternative empirical models, such as quantile regressions and regressions allowing for a more flexible relationship between the house price effects of bike sharing and the distance to subway stations.

\section{Quantile Regressions}

To investigate whether our findings are robust to houses at different price levels, following Peng and Chiang (2015), we adopt the approach of quantile regression (Koenker and Bassett, 1978). The dependent and independent variables are the same as in regression (1). We include houses in ordinary neighborhood that were transacted during the period 2011-2019. 
Table 7 Quantile regression results

\begin{tabular}{lllll}
\hline & $(1) \mathbf{Q 2 0}$ & $(2) \mathbf{Q 4 0}$ & $(3) \mathbf{Q 6 0}$ & $(4) \mathbf{8 0}$ \\
\hline InBike*Post & $-0.0115^{* * *}$ & $-0.0077^{* * *}$ & $-0.0070^{* * *}$ & $-0.0085^{* * * *}$ \\
InBike*DSub*Post & $(0.0022)$ & $(0.0018)$ & $(0.0019)$ & $(0.0019)$ \\
& $0.0065^{* * *}$ & $0.0051^{* * *}$ & $0.0046^{* * *}$ & $0.0038^{* * *}$ \\
Controls & $(0.0020)$ & $(0.0017)$ & $(0.0015)$ & $(0.0015)$ \\
\hline
\end{tabular}

Notes. We run quantile regression, using the simplex algorithm in the SAS software. The dependent and independent variables are the same as in regression (1). We include houses in ordinary neighborhoods that were transacted between 2011 and 2019. Numbers in parentheses are standard errors. The significant levels of $10 \%, 5 \%$, and $1 \%$ are marked with ${ }^{*},{ }^{* *}$, and ${ }^{* * *}$, respectively

Table 7 summarizes the coefficients of the two interaction terms that we are interested in. For each price quantile that we investigate, the coefficient of $\ln B i k e^{*}$ Post is significantly negative, and the magnitude is largest for the lowest price quantile. This indicates that the negative external effect of bike sharing is larger around cheaper houses. Moreover, the coefficient of $\ln B i k e^{*} D S u b^{*}$ Post is significantly positive for all the four quantiles. The magnitude is also larger for lower price quantiles, indicating that the positive value of bike sharing is higher for homeowners of cheaper houses. These results are understandable, to the extent that people living in cheaper houses tend to be more reliant on public transportation, and the quality of property management service there also tends to be lower.

\section{Using Distance Dummies Instead of DSub}

In the baseline regression, we interact the distance to the nearest subway station $(D S u b)$ with the usage intensity of shared bikes. To allow for a more flexible relationship between the house price effects of bike sharing and the distance to subway stations, now we use distance dummies instead of $D S u b$. More specifically, we define $D i s Q 2$ as a dummy that equals 1 for houses with a $D S u b$ between the $25^{\text {th }}$ percentile and the $50^{\text {th }}$ percentile, i.e. $\left(\mathrm{p} 25^{\text {th }}, \mathrm{p} 50^{\text {th }}\right]$, and 0 otherwise. Dis $Q 3$ equals 1 if the distance is within the range $\left(\mathrm{p} 50^{\text {th }}, \mathrm{p} 75^{\text {th }}\right.$ ], and 0 otherwise. DisQ4 equals 1 if the distance is above $\mathrm{p} 75^{\text {th }}$. Houses with a distance no more than the $25^{\text {th }}$ percentile are used as the benchmark. The $25^{\text {th }}, 50^{\text {th }}$, and $75^{\text {th }}$ percentiles correspond to the DSub values of $0.42 \mathrm{~km}, 0.63 \mathrm{~km}$, and $0.90 \mathrm{~km}$, respectively.

Then, replacing $D S u b$ with the vector (DisQ2, DisQ3, DisQ4), we repeat the baseline regression. We consider houses in ordinary neighborhoods transacted between 2011 and 2019. According to the (un-tabulated) results, the coefficient of $\ln B i k e^{*}$ Post is $-0.0070(\mathrm{p}=0.25)$. Though the level of statistical significance is not high, the coefficient's sign suggests that bike sharing negatively affect the house prices in areas close to subway stations. Moreover, the coefficients of $\ln$ Bike*DisQ2*Post, $\ln$ Bike*DisQ3*Post, and $\ln$ Bike*DisQ4*Post are 0.0004 $(\mathrm{p}=0.96), 0.0144(\mathrm{p}=0.10)$, and $0.0081(\mathrm{p}=0.32)$, respectively. Though not highly significant, they suggest that bike sharing positively affect house prices in areas with 
a relatively long distance from subway stations, but the positive effect declines when the distance becomes too long. This is understandable, because alternative transportation modes (e.g. bus) are more suitable for long-distance commuting. In general, these results are consistent with $\mathrm{H} 1$ and $\mathrm{H} 2$.

\section{Changes in the Subway Network During the Sample Period}

During our sample period 2011-2019, the route length of the subway network increased from 454 to $705 \mathrm{~km}$. But so far, the subway station locations used in this paper have been based on the POI data collected in March 2020. So it is possible that the "nearest station" of a house was not yet put into use when the house was transacted. Some may be concerned that this may introduce estimation bias on our major results. However, this should be not a big concern, because our sample area is relatively close to the downtown, where the subway network was mature and mostly constructed before 2011.

To formally address this concern, we hand collect the opening date of each subway station. Only 26 out of the 136 stations in our sample area were opened before 2011. We then do a subsample analysis. In particular, we drop a house $i$ transacted in year $t$ if its nearest station was opened in year $t$ or later. This leads to a sample size reduction of merely $4.6 \%$. We rerun the baseline regression with this subsample, using houses in ordinary neighborhoods. According to the (un-tabulated) results, the coefficient of $\ln B i k e^{*}$ Post is $-0.0102(\mathrm{p}=0.05)$, and the coefficient of $\ln B i k e^{*} D S u b^{*}$ Post is $0.0083(\mathrm{p}=0.01)$. They are consistent with our previous findings.

\section{Conclusion}

In this paper, we study the house price effects of bike sharing. We find that the introduction of bike sharing reduces the subway premium in house prices, and this result is driven by the neighborhoods where shared bikes are heavily used. This indicates that bike sharing generates a positive value by complementing the subway network. But meanwhile, bike sharing also has a negative price effect that may apply to all houses. The negative effect is weaker for houses in luxury neighborhoods and houses relatively close to city management teams, suggesting that it is caused by such problems as misplacement. In the vicinity of subway stations, where people can reach the stations by walking and the positive value of bike sharing as a complement to subways is virtually zero, the net house price effect of bike sharing is negative.

The above-mentioned effects of bike sharing on house prices have been strengthened by COVID-19. This is in line with the fact that bike sharing, as a public transportation mode that allows for social distancing, has earned a larger user base during the pandemic. As people become more confident about the long survival of the bike sharing industry, its house price effects also become more salient. But we should interpret the results related to COVID-19 with caution. Our sample period ends in July 2020, covering a post-pandemic period of merely seven months. Although we 
control for year-month fixed effects, it is still possible that other factors during this special period have contributed to the shifts in the relationship between bike sharing and house prices.

In addition, our findings from the subsample analysis has shown that the work of property management companies and city management teams eliminate not only the negative external effect, but also the positive value of bike sharing. This calls for a better approach to manage shared bikes. One possible way is creating a list of "correct" parking locations. Hellobike, a major competitor of Meituan Bike, is trying to do this in Songjiang District of Shanghai.

There are several possible extensions based on the present study. For example, we can investigate whether bike sharing leads to an increased supply of new houses in areas relatively far from subway stations. It is also meaningful to estimate the economic value of the environmental benefit of bike sharing. According to Mobike (2017), users of Meituan Bike had ridden more than 2.5 billion kilometers $(\mathrm{km})$ by April 2017, which is equivalent to a reduction of 540,000 tons of carbon emissions, or saving 460 million liters of gasoline. By improving air quality, bike sharing may increase local house prices.

\section{Appendix A: Variable definitions}

\begin{tabular}{|c|c|}
\hline Variable & Definition \\
\hline lnBike & $\begin{array}{l}\text { A measure of Meituan Bike usage intensity. It equals the natural logarithm of the number } \\
\text { of rides that start from a neighborhood during morning rush hours }(7: 30-9: 30) \text { or end at } \\
\text { it during evening rush hours (17:30-19:30) on } 9 \text { October } 2017\end{array}$ \\
\hline $\ln \operatorname{Prc}$ & Natural logarithm of house transaction price; the unit of transaction price is 10,000 yuan \\
\hline Post & $\begin{array}{l}\text { A dummy that equals } 1 \text { for the dates after the launch of Meituan Bike, and } 0 \text { for earlier } \\
\text { dates }\end{array}$ \\
\hline DSub & Distance to the nearest subway station $(\mathrm{km})$ \\
\hline Size & House size $\left(\mathrm{m}^{2}\right)$ \\
\hline Room & The number of bedrooms in a house \\
\hline Parlor & The number of parlors in a house \\
\hline Position & $\begin{array}{l}\text { The position of a house in the apartment building (underground/low/medium/high, cor- } \\
\text { responding to the integer } 1 / 2 / 3 / 4 \text { ) }\end{array}$ \\
\hline TotFloor & The number of floors in the apartment building \\
\hline Age & Building age \\
\hline ManaFee & The property management fee of a neighborhood (yuan $/ \mathrm{m}^{2} / \mathrm{month}$ ) \\
\hline Dcenter & Distance to the city center (i.e., People's Square) \\
\hline PostCOVID & $\begin{array}{l}\text { A dummy that equals } 1 \text { for the dates after the breakout of COVID-19, and } 0 \text { for earlier } \\
\text { dates }\end{array}$ \\
\hline
\end{tabular}

Acknowledgements We would like to thank the editor and an anonymous reviewer for very helpful and detailed comments that have led to a marked improvement of the paper. We thank Jeffrey Cohen, Siqi Zheng, and Vishal Gaur for helpful comments. We also thank the participants at 2020 ASSA (San 
Diego, U.S.), ZhiYuan Chua, Bo Zhao and other participants at the 2019 Conference on Financial Technology and Finance Development in China, held at Central University of Finance and Economics (Beijing, China), and the seminar participants at ShanghaiTech University (Shanghai, China), the International Workshop on Transportation Research held at University of International Business and Economics (Beijing, China), and the 2019 Conference of National Natural Science Foundation of China (Kunming, China) for useful suggestions. We are grateful to Meituan Technology Co., Ltd for providing the bike sharing data, and for the support of manager Xiang Tian, senior sustainability expert Hao Qin and director Qian Wang. This research is supported by National Natural Science Foundation of China [grant number: 71803110], Shanghai Sailing Program [grant number: 18YF1407800], and "Chen Guang" project of Shanghai Municipal Education Commission and Shanghai Education Development Foundation [grant number: 19CG41]. Partial financial support from the UBC Sauder Centre for Urban Economics and Real Estate is also gratefully acknowledged.

Authors' Contributions Equal contribution.

Funding This research is supported by National Natural Science Foundation of China [grant number: 71803110], Shanghai Sailing Program [grant number: 18YF1407800], and "Chen Guang" project of Shanghai Municipal Education Commission and Shanghai Education Development Foundation [grant number: 19CG41]. Partial financial support from the UBC Sauder Centre for Urban Economics and Real Estate is also gratefully acknowledged.

Availability of Data and Material We are grateful to Meituan Technology Co., Ltd for providing the bike sharing data, and for the support of manager Xiang Tian, senior sustainability expert Hao Qin and director Qian Wang. Other data used in this paper are collected from public source.

Code Availability Yes.

\section{Declarations}

\section{Conflicts of Interest No.}

\section{References}

Alonso, W. (1964). Location and Land Use: Towards a General Theory of Land Rent. Harvard University Press. Angrist, J. D., \& Pischke, J. S. (2009). Mostly Harmless Econometrics. Princeton University Press. https://doi.org/10.1515/9781400829828

Chu, J., Duan, Y., Yang, X., \& Wang, L. (2021). The last mile matters: Impact of dockless bike sharing on subway housing price premium. Management Science, 67(1), 297-316. https://doi.org/10.1287/ mnsc.2019.3550

Des Rosiers, F., Lagana, A., \& Theriault, M. (2001). Size and proximity effects of primary schools on surrounding house values. Journal of Property Research, 18(2), 149-168. https://doi.org/10.1080/ 09599910110039905

Eisenmann, C., Nobis, C., Kolarova, V., Lenz, B., \& Winkler, C. (2021). Transport mode use during the COVID-19 lockdown period in Germany: The car became more important, public transport lost ground. Transport Policy, 103, 60-67.

Gan, L., Yin, Z., Jia, N., Xu, S., Ma, S., \& Zheng, L. (2014). Data you need to know about China. Springer-Verlag.

Gu, T., Kim, I., \& Currie, G. (2019). To be or not to be dockless: Empirical analysis of dockless bikeshare development in China. Transportation Research Part a: Policy and Practice, 119, 122-147. https://doi.org/10.1016/j.tra.2018.11.007

Koenker, R., \& Bassett, G., Jr. (1978). Regression quantiles. Econometrica, 46(1), 33-50. https://doi.org/ $10.2307 / 1913643$

Li, H., Wei, Y. D., Wu, Y., \& Tian, G. (2019). Analyzing housing prices in Shanghai with open data: Amenity, accessibility and urban structure. Cities, 91, 165-179. https://doi.org/10.1016/j.cities. 2018.11.016 
Li, L., Ren, H., Zhao, S., Duan, Z., Zhang, Y., \& Zhang, A. (2017). Two dimensional accessibility analysis of metro stations in Xi'an, China. Transportation Research Part a: Policy and Practice, 106, 414-426. https://doi.org/10.1016/j.tra.2017.10.014

$\mathrm{Li}, \mathrm{T}$. (2020). The value of access to rail transit in a congested city: Evidence from housing prices in Beijing. Real Estate Economics, 48(2), 556-598. https://doi.org/10.1111/1540-6229.12222

Li, W., \& Joh, K. (2017). Exploring the synergistic economic benefit of enhancing neighbourhood bikeability and public transit accessibility based on real estate sale transactions. Urban Studies, 54(15), 3480-3499. https://doi.org/10.1177/0042098016680147

Mills, E. S. (1972). Urban Economics. Glenview: Scott, Foresman and Company.

Mobike. (2017). Bike-sharing and the City: 2017 White Paper. Beijing Mobike Technology Co., Ltd. Assessed on 21 February 2020, from: https://mobike.com/global/public/Mobike\%20-\%20White\% 20Paper\%202017_EN.pdf

Muth, R. F. (1969). Cities and Housing: The Spatial Pattern of Urban Residential Land Use. University of Chicago Press.

Pelechrinis, K., Zacharias, C., Kokkodis, M., \& Lappas, T. (2017). Economic impact and policy implications from urban shared transportation: The case of Pittsburgh's shared bike system. PLoS ONE, 12(8), e0184092. https://doi.org/10.1371/journal.pone.0184092

Peng, T. C., \& Chiang, Y. H. (2015). The non-linearity of hospitals' proximity on property prices: Experiences from Taipei. Taiwan. Journal of Property Research, 32(4), 341-361. https://doi.org/10. 1080/09599916.2015.1089923

Rossi-Hansberg, E., Sarte, P. D., \& Owens, R., III. (2010). Housing externalities. Journal of Political Economy, 118(3), 485-535. https://doi.org/10.1086/653138

Teulings, C. N., Ossokina, I. V., \& De Groot, H. L. F. (2018). Land use, worker heterogeneity and welfare benefits of public goods. Journal of Urban Economics, 103, 67-82. https://doi.org/10.1016/j.jue. 2017.10.004

Theisen, T., \& Emblem, A. W. (2018). House prices and proximity to kindergarten - costs of distance and external effects? Journal of Property Research, 35(4), 321-343. https://doi.org/10.1080/09599916. 2018.1513057

Theisen, T., \& Emblem, A. W. (2021). The road to higher prices: Will improved road standards lead to higher housing prices? The Journal of Real Estate Finance and Economics, 62(2), 258-282. https:// doi.org/10.1007/s11146-020-09751-y

Yin, J., Qian, L., \& Shen, J. (2019). From value co-creation to value co-destruction? The case of dockless bike sharing in China. Transportation Research Part d: Transport and Environment, 71, 169-185. https://doi.org/10.1016/j.trd.2018.12.004

Zhang, Y., Zhang, A., \& Wang, J. (2020). Exploring the roles of high-speed train, air and coach services in the spread of COVID-19 in China. Transport Policy, 94, 34-42. https://doi.org/10.1016/j.tranpol. 2020.05 .012

Zhou, Z., Chen, H., Han, L., \& Zhang, A. (2021). The effect of a subway on house prices: Evidence from Shanghai. Real Estate Economics, 49, 199-234. https://doi.org/10.1111/1540-6229.12275

Publisher's Note Springer Nature remains neutral with regard to jurisdictional claims in published maps and institutional affiliations. 